\title{
BIOMASS GROWTH IN UNSATURATED POROUS MEDIA: HYDRAULIC PROPERTIES CHANGES
}

\author{
I. BORSI, A. FARINA, A. FASANO, M. PRIMICERIO \\ Dipartimento di Matematica - Università di Firenze \\ Viale G. Morgagni, 67/A \\ 50134 Firenze, Italy \\ E-mail: borsi@math.unifi.it
}

\begin{abstract}
We present a model to describe the biomass growth process taking place in an unsaturated porous medium during a bioremediation process. We focus on the so-called column experiment. At the initial time biomass and polluted water is inoculated in the column. The subsequent changes of hydraulic properties are analyzed. We also show some preliminary simulations.
\end{abstract}

Keywords: Microbial growth; Porous media; Bioremediation.

\section{Introduction}

The effect of the microbial growth on hydraulic properties of porous media is a topic studied in the framework of many applications, e.g. oil recovery, wastewater treatment, bioremediation, etc. (see [1]).

Studies on flow through porous media in presence of biomass growth are presented in the papers by Rockhold et al. [2-4]. As stated there, additional work is needed for modelling unsaturated condition.

The objective of this study is thus to analyze the flow through a contaminated unsaturated porous medium in presence of biomass growth processes which induce changes in the hydraulic properties of the medium itself. In this paper we focus only on anaerobic processes, namely the model we develop does not account for the $\mathrm{O}_{2}$ consumption and diffusion.

\section{Problem description and physical assumptions}

We consider a vertical column (whose high is $L \sim 1 \mathrm{~m}$ ) of an unsaturated contaminated soil which represents a "laboratory scale" of a real vadose zone (the so-called "column experiment", see Fig. 1). The physical model is developed considering a 1-D approximation, so that $x$ denotes the vertical 


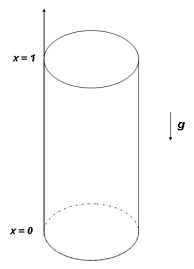

Fig. 1. A schematic of the column experiment.

coordinate of the column, pointing upwards.

At the initial time $(t=0)$ the saturation degree of the medium corresponds to the steady state. Then we inoculate biomass and (possibly) polluted water through the top surface. Our goal is to model the evolution of the biomass, pollutant concentration and hydraulic properties of the soil as well.

Hereafter we list the most significant physical assumptions (see also [5]):

A.1 The soil is a homogeneous, unsaturated, rigid porous medium.

A.2 The liquid phase which shares the empty space with air is composed by water (main component). We shall neglect the liquid density variation.

A.3 The pollutant is dissolved in water and adsorbed onto the soil grains. Moreover, the dissolved pollutant (below a certain concentration) acts as nutrient for the bacteria (bio-reduction), but above a certain threshold may become a toxic agent (see [6]).

A.4 The biomass is distributed either in water as suspension ("free biomass") or attached on the soil grains ("attached biomass"). In particular, there is no cluster formation in the free biomass. The mass of the single bacterium is known and denoted by $\mathfrak{m}_{b}$.

A.5 The attached biomass forms porous clusters so that the liquid can diffuse through them. The clusters porosity is a known constant denoted by $\varepsilon_{b}$. The pores are saturated at all times. Moreover, the concentration of the pollutant in the "entrapped water" equals the one of the "free water" (see [3], for instance). The number of bacteria that forms the unit mass of the attached biomass is a known constant denoted by $N^{\star}$, $\left[N^{\star}\right]=K g^{-1}$. Of course, $N^{\star}, \varepsilon_{b}$ and $\mathfrak{m}_{b}$ depend on the type of bacteria which are present inside the column.

A.6 We consider the attachment of free biomass on the clusters, but we neglect the inverse process (i.e. we neglect detachment). Indeed the 
experiments show that detachment is mainly due to the mechanical action caused by the "fast" water flux, [10].

A.7 The concentration of pollutant and bacteria in the liquid phase is low (few $p p m$ ).

Free biomass and attached biomass are responsible for different effects changing hydraulic properties. More precisely:

E.1 The free biomass causes essentially viscosity and surface tension variations.

E.2 The attached biomass growth causes medium porosity variations and affects the contact angle.

E.3 The above variations induce, in turn, changes in the permeability and in the relative saturation of the medium.

\section{Notations and basic equations}

We introduce the following quantities:

- $\varepsilon_{0},\left[\varepsilon_{0}\right]=[-]$, initial porosity of the column (known parameter).

- $\varepsilon_{b},\left[\varepsilon_{b}\right]=[-]$, clusters porosity (considered a known constant).

- $\phi^{f}$, volume fraction occupied by the liquid and the gaseous (air) phase.

- $\phi^{c}$, volume fraction occupied by the clusters.

- $\sigma,[\sigma]=[-]$ liquid phase saturation.

- $\theta_{l m}=\frac{\text { volume of "mobile" liquid }}{\text { porous medium volume }},\left[\theta_{l m}\right]=[-]$. In particular, $\theta_{l m}=\sigma \phi^{f}$.

- $\theta_{l b}=\varepsilon_{b} \phi^{c}=\frac{\text { volume of "clusters }- \text { stored" liquid }}{\text { porous medium volume }},\left[\theta_{l b}\right]=[-]$.

We thus have that

$$
\phi^{f}+\phi^{c}=\varepsilon_{0} .
$$

So, the volume fraction occupied by the liquid (accounting for mobile and stored) is

$$
\theta_{l m}+\theta_{l b}=\sigma \phi^{f}+\varepsilon_{b} \phi^{c}=\left(\sigma-\varepsilon_{b}\right) \phi^{f}+\varepsilon_{b} \varepsilon_{0} .
$$

The dependent variables which have to be determined are:

- $\sigma(x, t)$, the liquid phase saturation.

- $\phi^{c}(x, t)$, or alternatively $\phi^{f}=\varepsilon_{0}-\phi^{c}$.

- $N_{l}(x, t)=\frac{\text { number of free bacteria in the liquid phase }}{\text { unit mass of free liquid }},\left[N_{l}\right]=K g^{-1}$.

- $w_{A}(x, t)=\frac{\text { mass of pollutant dissolved in the liquid phase }}{\text { unit mass of free liquid }}$. 
- $w_{s}(x, t)=\frac{\text { mass of pollutant adsorbed onto soil grains }}{\text { unit mass of solid matrix }}$.

\section{Darcy's law and Richards equation}

We define the water pressure $P$ and introduce the capillary pressure $P_{c}$ and the pressure head $\psi$, setting

$$
P_{c}=P_{\text {air }}-P=-P, \quad \psi=\frac{-P}{\rho g}=\frac{P_{c}}{\rho g},
$$

since, as usual, $P_{\text {air }}$ has been rescaled to 0 .

The well-known Darcy's law describes the specific discharge $q$,

$$
q=-\frac{\rho g \mathcal{K}_{\text {sat }}}{\mu} k_{r e l}\left(\frac{\partial \psi}{\partial x}+1\right)
$$

where $\rho$ water density, $g$ gravity acceleration and

- $\mathcal{K}_{\text {sat }}=\mathcal{K}_{\text {sat }}\left(\phi^{f}\right)$, saturated permeability, $\left[\mathcal{K}_{\text {sat }}\right]=m^{2}$. For instance, we mention the Kozeny-Carman formula [11].

- $k_{\text {rel }}=k_{\text {rel }}(\psi),\left[k_{r e l}\right]=[-]$, relative permeability (see [14]).

- $\mu$ is the liquid phase viscosity. We assume the following law (based on experimental observations [2])

$$
\mu=\tilde{\mu}\left(N_{l}\right)=\mu_{0}\left(1+b_{3} N_{l}\right),
$$

with $b_{3}$ empirical parameter and $\mu_{0}$ water viscosity (i.e. the liquid viscosity in absence of biomass).

Next, the Richards' equation [14] reads as

$$
\frac{\partial}{\partial t}\left[\theta_{l m}+\theta_{l b}\right]=-\frac{\partial q}{\partial x}
$$

Now, introducing the saturation curve $\sigma=\tilde{\sigma}(\psi)$, we have $\theta_{l m}=\phi^{f} \tilde{\sigma}(\psi)$ so that, exploting (1), the mass conservation (3) rewrites as

$$
\frac{\partial}{\partial t}\left[\left(\sigma-\varepsilon_{b}\right) \phi^{f}\right]=-\frac{\partial q}{\partial x} .
$$

Evolution equation for the attached biomass phase

According to the literature (see for instance, [7] $\$ 2.3$ and [12]) we set

$$
\begin{gathered}
\frac{\partial \phi^{c}}{\partial t}=\underbrace{\left(c_{1} N^{\star}\right)\left[\varepsilon_{0} f\left(w_{A} \varepsilon_{b} \phi^{c}\right)-\phi^{c}\right] \phi^{c}}_{\text {biomass "bulk growth" }} \\
+\underbrace{\lambda \mathfrak{H}\left(w_{s}-w_{A}\right) N_{l} \theta_{l m}}_{\text {attachment }},
\end{gathered}
$$


where:

- $\left[c_{1}\right]=K g s^{-1}$.

- $w_{A} \varepsilon_{b} \phi^{c}$ is the amount of nutrient available for the attached biomass.

- $\varepsilon_{0} f\left(w_{A} \varepsilon_{b} \phi^{c}\right)$, is a modified form of the carrying capacity (see [13] $\left.\S 1.2\right)$. Actually, $\varepsilon_{0}$ is the maximum volume fraction allowed for the attached biomass and it is "modulated" by the function $f, 0 \leq f \leq 1$, which accounts of both amount of nutrient and toxic effects (see assumption A.3).

- $\mathfrak{H}(\cdot)$ is the Heaviside function. We notice that the attachment term could be multiplied also by a function of $\phi^{c}$, i.e. an "effective surface" term modeling the so-called collector and collision (or sticking) efficiencies.

\section{Evolution equation for the free biomass}

The free biomass is a component of the liquid phase. Therefore, following $[8,9]$, the evolution equation for $N_{l}$ is

$$
\begin{aligned}
\frac{\partial}{\partial t}\left(N_{l} \theta_{l m}\right)= & \underbrace{-\frac{\partial}{\partial x}\left[q N_{l}\right]+\frac{\partial}{\partial x}\left[a_{L}|q| \frac{\partial N_{l}}{\partial x}\right]}_{\text {advection/dispersion }} \\
+ & \underbrace{c_{1}\left[N_{\max } f\left(w_{A}\right)-N_{l}\right] N_{l} \theta_{l m}}_{\text {free biomass growth }} \\
& -\underbrace{\lambda \mathfrak{H}\left(w_{s}-w_{A}\right) N_{l} \theta_{l m}}_{\text {attachment }} .
\end{aligned}
$$

The first term in (6) is the divergence of the advective flux $\mathbf{J}_{a d v}=-q N_{l}$. The second term represents the dispersive flux $\mathbf{J}_{\text {disp }}$, which in 1-D setting has the following form

$$
\mathbf{J}_{\text {disp }}=-\sigma \phi^{f} D, \quad D=\frac{a_{L}|q|}{\sigma \phi^{f}}
$$

with $a_{L}$ longitudinal dispersion coefficient (see [14]).

The "bulk growth" of the free biomass is modeled as in the case of the attached biomass, i.e. by means of a logistic-type dynamics. Here $N_{\max }$ is the equilibrium value. We set

$$
N_{\max }=\mathfrak{n} N^{\star}, \quad 0<\mathfrak{n}<1 .
$$


Equation for the adsorbed pollutant.

We describe the dynamics of $w_{s}$ by the following equation

$$
\begin{aligned}
\frac{\partial w_{S}}{\partial t}= & \underbrace{h_{A} w_{A} \varepsilon_{b} \phi^{c}\left(w^{\star}-w_{S}\right)}_{\text {adsorption term }} \\
& -\underbrace{h_{D B} N^{\star} \phi^{c} w_{S}}_{\text {bio-reduction term }},
\end{aligned}
$$

stipulating, essentially, that only two effects are important: adsorption (desorption) and bio-reduction. In particular,

- $w_{A} \varepsilon_{b} \phi^{c}=\varepsilon_{b} \theta_{l b}$ is the amount of pollutant dissolved inside the biomass clusters.

- $w^{\star}$ is maximum concentration of pollutant (known parameter) which can be adsorbed by the soil.

- $h_{A},\left[h_{A}\right]=s^{-1}$, is the adsorption/desorption rate per unit concentration.

- $h_{D B},\left[h_{D B}\right]=K g s^{-1}$, bio-reduction rate per unit mass.

Equation for $w_{A}$.

The total amount of pollutant (per unit volume of porous medium) dissolved in the free and "entrapped" water is $w_{A}\left(\theta_{l m}+\theta_{l b}\right)$, i.e. $w_{A}\left(\theta_{l m}+\varepsilon_{b} \phi^{c}\right)$. According to [9], we write for it the following equation

$$
\begin{aligned}
& \frac{\partial}{\partial t}\left[w_{A}\left(\theta_{l m}+\varepsilon_{b} \phi^{b}\right)\right]=-\underbrace{\frac{\partial}{\partial x}\left[q w_{A}\right]+\frac{\partial}{\partial x}\left[a_{L}|q| \frac{\partial w_{A}}{\partial x}\right]}_{\text {advection/dispersion term }} \\
&-\underbrace{h_{A} w_{A} \varepsilon_{b} \phi^{c}\left(w^{\star}-w_{S}\right)}_{\text {adsorption term }} \\
&-\underbrace{h_{D B}\left[\theta_{l m} N_{l}+\phi^{c} N^{\star}\right] w_{A}\left(\theta_{l m}+\varepsilon_{b} \phi^{c}\right)}_{\text {bio-reduction term }} .
\end{aligned}
$$

The bio-reduction term in (8) depends on the amount of bacteria which are present either in the liquid and in the clusters.

Summarizing, we have to solve the system of the governing equations (4), (5), (6), (7) and (8), which has to be endowed with initial and boundary conditions. 


\section{A simplified approach: biofilm and fluid media scaling}

We now illustrate the basic idea of an approach to simplify the problem. The key point is to consider a porous medium constituted by a network of capillary tubes distributed uniformly in space. Next, we assume that the attached biomass phase forms a uniform layer (biofilm) completely coating the internal surfaces of the capillary tubes.

Focusing now on a single capillary tube, we compare two scenarios: capillary tube partially filled with "pure" water and capillary tube whose walls are coated by the biofilm and partially filled by a liquid whose components are water, bacteria and pollutant.

Denoting by $p_{c}$ and $p_{c, \text { bio }}$ the capillary pressures which refer to the above scenarios, we may write the following Laplace formulas

$$
p_{c}=\frac{2 \gamma \cos \alpha}{R}, \quad p_{c, b i o}=\frac{2 \gamma_{b i o} \cos \left(\alpha_{b i o}\right)}{R_{b i o}},
$$

where $R$ and $R_{b i o}$ denote the capillary radii, $\gamma$ and $\gamma_{b i o}$ are the surface tensions and $\alpha, \alpha_{b i o}$ are the contact angles. Therefore,

$$
\frac{p_{c, b i o}}{p_{c}}=\frac{\gamma_{b i o}}{\gamma} \frac{\cos \alpha_{b i o}}{\cos \alpha} \frac{R}{R_{b i o}}
$$

We now assume that the above formula holds true also for the averaged quantities, i.e.

$$
\frac{P_{c, b i o}}{P_{c}}=\frac{\gamma_{b i o}}{\gamma} \frac{\cos \alpha_{b i o}}{\cos \alpha} \frac{\langle R\rangle}{\left\langle R_{b i o}\right\rangle},
$$

where $P_{c, b i o}=\left\langle p_{c, b i o}\right\rangle, P_{c}=\left\langle p_{c}\right\rangle$, since $\langle\cdot\rangle$ denotes the R.E.V. average. Now, selecting appropriate constitutive equations ${ }^{\mathrm{a}}$ for

$$
\frac{\cos \alpha_{b i o}}{\cos \alpha}, \frac{\gamma_{b i o}}{\gamma} \text { and } \frac{\langle R\rangle}{\left\langle R_{b i o}\right\rangle},
$$

we can define the parameter $\beta=\beta\left(\phi^{f}, N_{l}\right)$, (called scaling factor for the capillary pressure), such that

$$
\frac{P_{c, b i o}}{P_{c}}=\beta\left(\phi^{f}, N_{l}\right), \Rightarrow \psi=\beta\left(\phi^{f}, N_{l}\right) \psi_{0},
$$

where $\psi_{0}=-\frac{P_{c}}{\rho g}$ is the pressure head in absence of biomass. Therefore,

$$
\theta_{l m}=\tilde{\sigma}\left(\beta\left(\phi^{f}, N_{l}\right) \psi_{0}\right)\left(\varepsilon_{0}-\phi^{c}\right)
$$

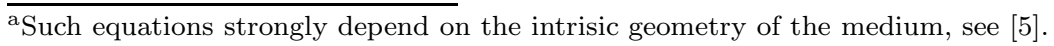


Such an approach (often called fluid media scaling, see [15]), presents an evident advantage: once the flow problem is specified, we determine $\psi_{0}$ using the "classical" Richards' equation, i.e. equation (4) where the term due to porosity changes is absent. As second step we evaluate $\psi$ exploiting (10) and $q$ through (2). Therefore, the mathematical problem is strongly simplified and its numerical solving turns out easier. Of course, the fluid media scaling suffers from an evident drawback: $\psi$ and $q$ do not fulfill the Richards' equation, i.e. mass conservation. Hence, such a property needs to be tested a posteriori.

\section{Numerical simulations}

In this section we present few numerical simulations we worked out considering the simplified model. A deeper analysis of the results can be found in the forthcoming paper [5]. Our main goals are: (i) to show that the found solution satisfies (within a suitable tolerance) the Richards' equation; (ii) to show that the results obtained agree, at least qualitatively, with the experimental data; (iii) to put in evidence that, in certain circumstances, variation of porosity and hydraulic properties is significant.

\subsection{Problem setting}

The PDEs system was solved in a 1D domain. Concerning the soil, we used the well-known van-Genuchten and Mualem forms for the saturation and permeability curve, respectively (see [14]). Moreover, a Cozeny-Karman function for the saturated permeability $k_{\text {sat }}\left(\phi^{f}\right)$ has been selected (see [11]). Finally, we run the simulation considering $T_{\max }=7$ day as maximum time of the process.

\section{Initial conditions}

The initial stage of the experiment is characterized by absence of attached biomass and a given pollutant concentrations. Hence we set

$$
w_{A}(x, 0)=w_{A}^{0}, \quad w_{S}(x, 0)=w_{S}^{0}, \quad N_{l}(x, 0)=0, \quad \phi^{c}(x, 0)=0,
$$

with $w_{A}^{0}=0.3$ and $w_{S}^{0}=0.7$.

\section{Boundary conditions}

Following [4] we set on the column bottom, $x=0$,

$$
\left.\frac{\partial N_{l}}{\partial x}\right|_{x=0}=0,\left.\quad \frac{\partial w_{A}}{\partial x}\right|_{x=0}=0 .
$$


On the column top, $x=1$ water (with pollutant and bacteria) is inoculated in the medium. We stipulate that pollutant and bacteria concentration in the inflow water are known, hence,

$$
N_{l}(1, t)=N_{1}(t), \quad w_{A}(1, t)=w_{A 1}(t) .
$$

\subsection{Simulation results}

First, we mention the computed value of the quantity

$$
\max _{(x, t) \in[0,1] \times\left[0, t_{c}\right]}\left|\frac{\partial\left(\theta_{l m}+\theta_{l b}\right)}{\partial t}+\frac{\partial q}{\partial x}\right| \sim 3.8 \times 10^{-7}
$$

which definitively shows that the computed solution $\psi=\beta \psi_{0}$ satisfies the Richards' equation.

Moreover, in Fig. 5.2-5.2 we report the plot of the most sgnificant quantities computed during the simulation. All the values are plotted at initial, intermedium and final time step.

Further comments on the obtained results will be reported in the forthcoming work [5].

\section{References}

1. Holden P.A., Fierer N., Microbial processes in the vadose zone, Vadose Zone J. 4 (2005), 1-21.

2. Rockhold M.L., Yarawood R.R., Niemet M.R., Bottomley P.J., Selker J.S., Consideration for modelling bacterial-induced changes in hydraulic properties of variably saturated porous media, Adv. Wat. Res. 25 (2002), 477-495.

3. Rockhold M.L., Yarwood R.R., Seker J.S., Coupled microbial and transport processes in soils, Vadose Zone J. 3 (2004), 368-383.

4. Rockhold M.L., Yarawood R.R., Niemet M.R., Bottomley P.J., Selker J.S., Experimental observations and numerical modeling of coupled microbial and transport processes in variably saturated sand, Vadose Zone J. 4 (2005), 407417.

5. Borsi I., Farina A., Fasano A., Primicerio M., Modelling changes of hydraulic properties induced by biomass growth in unsaturated porous media, to appear.

6. Gallo C., Hassanizadeh S.M., Modeling NAPL dissolution and biodegradation interactions: effect of toxicity and biomass growth limitations, Computational Methods in Water Resources, S.M. Hassanizadeh et al. (Eds.), vol. 1 (2002), 859-866, Elsevier, Amsterdam.

7. Rajagopal K.R., Tao L., Mechanics of Mixtures, World Scientific, Singapore, 1995.

8. Schäfer A. et al., Transport of bacteria in unsaturated porous media, J. Contam. Hydrol. 33 (1998), 149-169. 
9. Hassanizadeh S.M., Derivation of basic equations of mass transport in porous media, Part 2. Generalized Darcy's and Fick's laws, Adv. Water Resources, 9, 207-222 (1986)

10. MacDonald T.R., et al., Effects of shear detachment on biomass growth and in situ bioremediation, Ground Water, 37 (1999), 555-563.

11. Carman, P.C, Fluid flow through granular beds, Transactions of Chemical Engineering, 15, 150-166, London (1937).

12. Kingsland, S.E., Modeling Nature, University of Chicago press, Chicago, 1985.

13. Brauer, F., Castillo-Chavez, C., Mathematical Models in Population Biology and Epidemiology, Texts in Applied Mathematics, Vol. 40, Springer, New York, 2001.

14. Bear J., Verruijt A., Modelling Groundwater Flow and Pollution, Theory and Applications of Transport in Porous Media, Bear J. (Ed.), vol. 1, Kluwer, Dordrecht, 1987.

15. Miller, E.E., Miller R.D., Physical theory for capillary flow phenomena, J. Appl. Phys., 27 (1956), 324-332.

16. Coirier J., Mécanique des Milieux Continus, Dunod, Paris 1997.

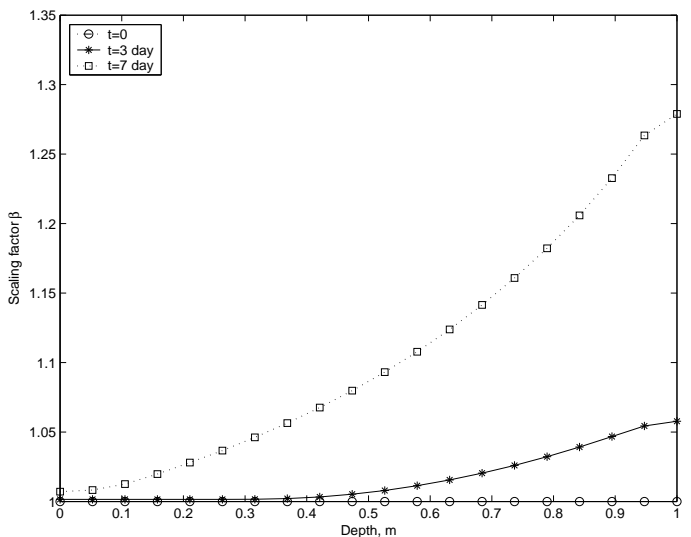

Fig. 2. Plot of the scaling factor $\beta$ at different time values. 


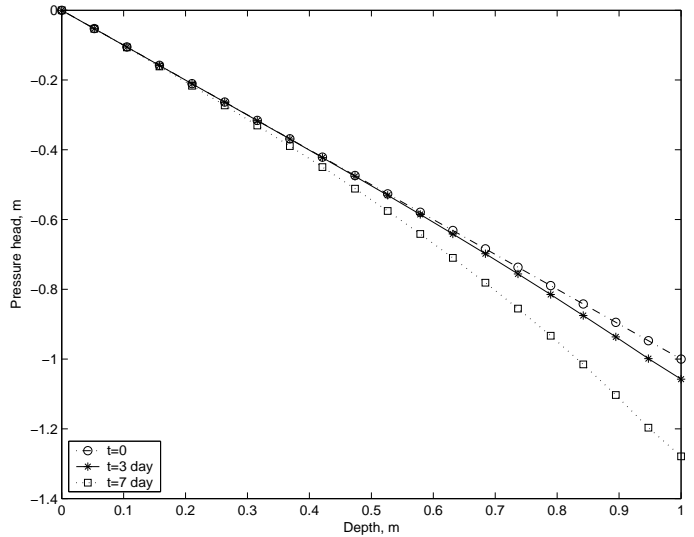

Fig. 3. Pressure head $\psi$ at different times.

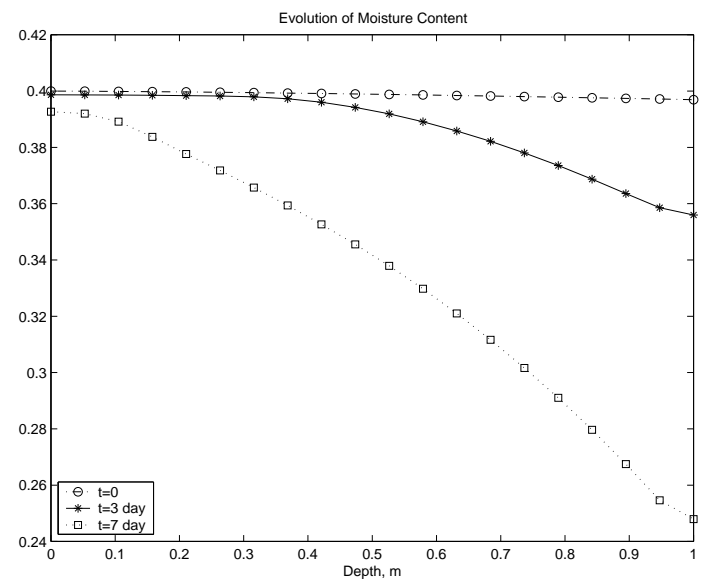

Fig. 4. Moisture content $\left(\theta_{l m}+\theta_{l b}\right)$ at different times.

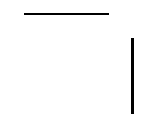




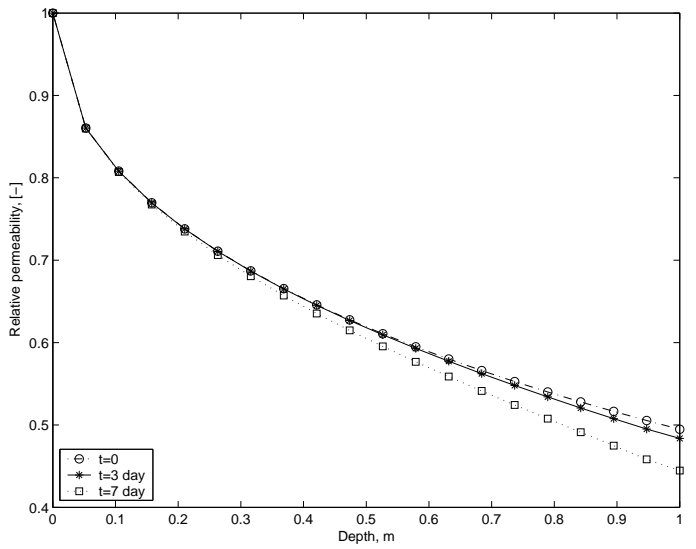

Fig. 5. Relative permeability $k_{r e l}$ at different times. 